\title{
List of Suppliers of Specialized Items
}

\author{
Axon Instruments \\ 1101 Chess Drive \\ Foster City, CA 94404 (USA) \\ Tel. +1 4155719400 \\ Fax +1415 5719500 \\ In Germany: 0130810458 \\ In Switzerland: 046057323 \\ (electrophysiological equipment, patch \\ clamp amplifiers, switch clamp \\ amplifiers)
}

Becton-Dickinson Labware (Falcon) 1950 Willaim Drive

Oxnard, CA 93030 (USA)

Becton Dicknson GmbH

Postfach 101629

D-69006 Heidelberg (Germany)

Tel. +4962213050

Fax +496221303609

(cell culture equipment)

Biermann GmbH (DPC)

Hohe Strasse 4-8

D-61231 Bad Nauheim (Germany)

Tel. +49 603299400

Fax + 496031994200

(antibodies)

Biologic

Rue de l'Europe 1

F-38640 Claix (France)

Tel. + 3376986831

Fax +3376 986909

(electrophysiological equipment, pullers, patch clamp amplifiers)

Biometra

Rudolf-Wissell Strasse 30

D-37079 Göttingen (Germany)

Tel. +49551506860
Fax +495515068666

550 N. Reo Street \#101

Tampa, FL 33609 (USA)

Tel. +1 8132878815

Fax +1 8132821936

(labware, SDS-PAGE equipment)

Boehringer Mannheim

Sandhofer Strasse 116

D-68305 Mannheim (Germany)

Tel. +496217590

Fax +496217592890

(biochemistry, signal transduction reagents, immunochemistry)

Chemicon

28835 Single Oak Drive

Temecula, CA 92590 (USA)

Tel. + 19096768080

Fax +19096769209

(antibodies)

Clark Electromedical Instruments

PO Box 8

Pangbourne, Reading RG8 7HU (UK)

Tel. + 441734843888

Fax +44 1734845374

(electrophysiological equipment)

Dianova

Postfach 301250

D-20305 Hamburg (Germany)

Tel. +4940450670

Fax + 494045067390

(antibodies)

FMI Föhr Medical Instruments

In der Grube 13

D-64342 Seeheim/Ober-Beerbach

(Germany) 
Tel. +496257962260

Fax + 496257962262

(electrophysiological equipment, pullers, patch clamp amplifiers)

Gibco/BRL

Life Technologies

Dieselstrasse 5

D-76344 Eggenstein (Germany)

Tel. +49 130830902

or: Gibco Ltd

PO Box 35

Paisley PA3 4EF (UK)

(cell culture media, biochemica and equipment)

Greiner Labortechnik

Maybachstrasse 2

D-72636 Frickenhausen (Germany)

(plastic cell culture flasks, cell culture equipment)

Hameg

Kelsterbacher Strasse 15-19

D-60528 Frankfurt/Main (Germany)

Tel. +4969678050

Fax +49696780513

(oscilloscopes)

Heka Elektronik

Wiesenstrasse 71

D-67466 Lambrecht (Germany)

Tel. +4963258036

Fax + 4963258039

(electrophysiological equipment, pullers, patch clamp amplifiers)

Heraeus Instruments $\mathrm{GmbH}$

Postfach 1563

D-63405 Hanau (Germany)

Tel. +49618135413

Fax +49618135739

(cell culture equipment, incubators, benches)
Hewlett-Packard

Rothebühlstrasse 81

D-70197 Stuttgart (Germany)

Tel. +49711619650

Fax +4971161965 50

(oscilloscopes, recorders)

Immunotech

Luminy case 915

F-13288 Marseille Cedex 9 (France)

Postfach 101526

D-20010 Hamburg (Germany)

Tel. +4940322180

Fax +4940323969

(antibodies)

Integra Biosciences

Tecnomara GmbH

Ruhberg 4

D-35461 Fernwald (Germany)

Tel. +4964048090

(cell culture equipment)

Leica Vertrieb GmbH

Lilienthalstrasse 39-45

D-64606 Bensheim (Germany)

Tel. +496251 1360

Fax + 496251136155

(microscopes and equipment)

List Elektronic

Pflungstädter Strasse 18-20

D-64297 Darmstadt (Germany)

Tel. +49 615156000

Fax +49 615156060

(electrophysiological equipment, pullers, patch clamp amplifiers)

Luigs \& Neumann

Boschstrasse 19

D-40880 Ratingen (Germany)

Tel. +49 2102442035

Fax +49 2102442036

(micromanipulators and setups) 
Märzhäuser Wetzlar GmbH \& Co KG

An den Fichten 35

D-35579 Wetzlar (Germany)

Tel. +49644191160

Fax +496441911640

(micromanipulators)

Merck

Frankfurter Strasse 250

D-64293 Dramstadt (Germany)

Tel. +496151720

Fax +49615172 2000

(biochemica)

Millipore GmbH

Hauptstrasse 87

D-65760 Eschborn (Germany)

Tel. +4961964940

Fax +49619643901

or: Millipore Corporation

8 Ashby Road

Bedford, MA 01730 (USA)

(filter technique, cell biology)

Narishige

Unit 7 Willow Business Park, Willow Way London SE2 64QP (UK)

Tel. +441816998282

Fax +441816998299

(electrophysiological equipment, pullers, micromanipulators)

Nikon

Tiefenbroicher Weg 25

D-40472 Düsseldorf (Germany)

Tel. +4921194140

Fax +492119414330

(microscopes and equipment)

NPI electronic

Häldenstrasse 62

D-71732 Tamm

Tel. +497141601534

Fax +497141601266

(electrophysiological equipment, patch clamp amplifiers, switch clamp amplifiers)

\section{Nunc GmbH}

Hagenauer Strasse 21a

D-65203 Wiesbaden-Biebrich (Germany)

Tel. +4961167095

Fax +49611607348

or: 2000 Aurora Road

Naperville, IL 60566 (USA)

(cell culture equipment)

Olympus Optical GmbH \& Co.

Wendenstrasse 14-19

D-20097 Hamburg (Germany)

Tel. +49 40237730

Fax + 494023773647

(microscopes, microforges and equipment)

Pacer Scientific Instruments

5649 Valley Oak Drive

Los Angeles, CA 90068 (USA)

Tel. + 12134620636

Fax +12134621430

(recorder, micromanipulators, glass capillaries, puller, stimulators, amplifiers)

Sarstedt

Postfach 1220

D-51582 Nümbrecht (Germany)

Tel. +4922933050

(cell culture equipment, plastic culture flasks)

Sartorius AG

D-37070 Göttingen (Germany)

Tel. +495513080

(filters for cell cultures, labware)

Science Products

Hofheimer Strasse 63

D-65719 Hofheim (Germany)

Tel. +4961925046

Fax +4961925053

(electrophysiological equipment, pullers, patch clamp amplifiers) 


\author{
Sigma Chemie \\ Grünwalder Weg 30 \\ D-82039 Deisenhofen (Germany) \\ Tel. +4901305155 \\ Fax +4901306490 \\ or: Sigma Chemical Co. \\ PO Box 14508 \\ St. Louis, MO 63178 (USA)
}

(chemicals, antibodies, cell culture

equipment, labware)

Sutter Instruments

40 Leveroni Court

Novato, CA 94949 (USA)

Tel. + 14158830128

Fax +14158830572

(electrophysiological equipment, pullers, patch clamp amplifiers)

\section{Tektronix}

Colonia Allee 11

D-51067 Köln (Germany)

Tel. +49221969690

Fax +4922196969362

(oscilloscopes)
Warner Instrument Corp.

1125 Dixwell Avenue

Hamden, CT 06514 (USA)

Tel. +1 2037760664

Fax + 12037761278

(glass, AD/DA converter, amplifiers and equipment)

WPI World Precision Instruments

Harry Fein

Liegnitzer Strasse 15 D-10999 Berlin

(Germany)

Tel. +4930618 8845

Fax +4930618 8670

(microscopes, oscilloscopes and electrophysiological equipment)

Carl Zeiss Jena

Tatzendpromenade 1a

D-07740 Jena (Germany)

Tel. +493641 642420

Fax +49 3641643140

(microscopes, micromanipulators and equipment)

The items which may be of interest for gap junction research as outlined in the book are given in brackets. This is, however, not the entire product list of the supplier. It was not possible to incorporate all companies which supply items in the various fields of research. This is a list of suppliers mentioned somehow in this book and is not intended to represent a complete list of suppliers for cell culture techniques, electrophysiology, biochemistry and labware. 